\title{
Variations
}

Variations

Revue internationale de théorie critique

11 | 2008

La beauté est dans la rue

\section{Freud : guerre et Kultur}

\section{David Benhaim}

\section{(2) OpenEdition \\ Journals}

Édition électronique

URL : http://journals.openedition.org/variations/269

DOI : 10.4000/variations.269

ISSN : 1968-3960

\section{Éditeur}

Les amis de Variations

\section{Référence électronique}

David Benhaim, «Freud : guerre et Kultur », Variations [En ligne], 11 | 2008, mis en ligne le 01 février 2012, consulté le 19 avril 2019. URL : http://journals.openedition.org/variations/269 ; DOI : 10.4000/ variations.269

Ce document a été généré automatiquement le 19 avril 2019

Les ami•e•s de Variations 


\title{
Freud : guerre et Kultur
}

\author{
David Benhaim
}

\section{NOTE DE L'ÉDITEUR}

Première publication sur www.theoriecritique.com, « La beauté est dans la rue », 1er mai 2008, pp. 100-112

\section{a/ Les analystes et les écrits sur la Kultur}

1 Les écrits de Freud sur la Kultur sont, en général, aujourd'hui oubliés des analystes. Ils les voient comme des écrits marginaux où il se serait amusé à appliquer la méthode psychanalytique à des problèmes qui l'intéressaient ou le préoccupaient. Ils n'auraient, à leurs yeux, que peu de pertinence psychanalytique, étant plus près de la spéculation philosophique que de la vraie psychanalyse. Qu'est-ce que, selon eux, la vraie psychanalyse ? Celle qui reste collée à la clinique, reconstruit le déroulement d'une cure, mais finit souvent par s'engluer dans la professionnalisation, comme l'avait fait remarquer Adorno avec beaucoup d'inquiétude; l'autre, celle qui spécule sur la Kultur et ses symptômes n'intéresse pas les vrais cliniciens. A quoi bon lire des textes dont on ne voit pas la portée clinique ? s'exclament souvent ces analystes dont la myopie intellectuelle n'est pas le seul défaut.

2 Ce faux partage aboutit à distinguer un Freud clinicien, vrai psychanalyste, d'un Freud penseur, clivé du premier, qui, à ses heures, spécule sur les phénomènes anthropologiques et culturels. Il est fondé sur une méconnaissance du lien entre les écrits cliniques et ceux qui portent sur la Kultur. Pourtant Freud a toujours affirmé l'idée d'un antagonisme entre la Kultur et la vie pulsionnelle; dans ses préoccupations intellectuelles, cela remonte aussi loin que les Lettres à Fliess. Dans un manuscrit du 31 mai 1897, il lui écrivait que l'inceste est un fait antisocial auquel, pour exister, la civilisation a dû peu à peu renoncer ${ }^{1}$. Il a toujours soutenu sans se dédire que la névrose est un symptôme de la Kultur. Dans son article, La morale sexuelle civilisée, il écrit : Je dois, au contraire, attirer l'attention sur le fait que la névrose, où qu'elle porte et quel que soit celui chez qui on la rencontre sait faire 
échouer le dessein civilisateur ${ }^{2}$. Si nous jetons un regard sur ses derniers écrits, nous pouvons constater qu'une œuvre comme Malaise dans la culture repose sur l'idée que le destin de l'individu et celui de la communauté sont indissociables, l'un se jouant à travers l'autre. Les écrits sur la guerre ne feront qu'illustrer cette idée.

3 Pourquoi la guerre? appartient à ce genre d'écrits anthropologiques qui, comme Malaise dans la culture, L'avenir d'une illusion, L'Homme Moïse et la religion monothéiste, s'interroge sur le devenir de cette Kultur. Avec Actuelles sur la guerre et la mort ${ }^{3}$ de 1915, ce texte constitue les réflexions de Freud sur la guerre.

\section{b/ La fin d'une illusion} très peu de moralité dans leurs relations, alors qu'à l'intérieur, ils s'étaient montrés les champions des normes éthiques; d'autre part, des individus qui, comme participants à la culture humaine la plus élevée, avaient toujours fait preuve de respect et de compréhension les uns à l'égard des autres, se sont livrés, dans leur conduite guerrière, à des manifestations de brutalité auxquelles on ne se serait jamais attendu de leur part.

11 Alors que Valéry et Zweig interrogent les idéaux de la culture du point de vue de leur valeur civilisatrice, Freud les interrogera du point de vue de l'économie psychique; Valéry parlera de crise, alors que Freud parlera plus tard de malaise ${ }^{6}$; cependant, les trois réagiront devant le constat de l'effondrement des idéaux de l'homme occidental et du tissu communautaire européen [...] La désillusion est infligée par la culture ${ }^{7}$, comme le souligne Laurence Kahn. Ce malaise ne s'apaisera pas, mais ira en grandissant jusqu'à accomplir une régression vers une barbarie presque préhistorique ${ }^{8}$. C'est dans ce contexte que l'interrogation sur la guerre surgit. 
12 Dans son introduction ${ }^{9}$ à Pourquoi la guerre ?, James Strachey nous explique les circonstances dans lesquelles prit naissance la correspondance entre Freud et Einstein. En 1931, la Commission permanente pour la littérature et les arts de la Société des Nations charge l'Institut International de Coopération intellectuelle d'organiser un échange épistolaire entre des intellectuels représentatifs «sur des thèmes choisis pour servir aux intérêts communs de la Société des Nations et de la vie intellectuelle.» L'Institut s'adresse à Einstein qui, à son tour, suggère Freud comme interlocuteur. En juin 1932, le secrétaire de l'Institut écrit à Freud pour l'inviter à participer à cet échange. En août de la même année, Einstein envoie sa missive à Freud qui répond un mois plus tard. L'année suivante, 1933, année fatidique pour l'Europe, l'Institut publie cette correspondance à Paris en trois langues - allemand, français et anglais, sous le titre, Pourquoi la guerre ?, mais l'Allemagne interdit sa diffusion sur son territoire.

\section{La lettre d'Einstein}

Curieusement les analystes ont accordé très peu d'attention à la lettre d'Einstein et n'ont parfois publié que la seule réponse de Freud ${ }^{10}$. Pourtant, comme nous le verrons, cette réponse ne se comprend que par rapport aux questions que lui pose son interlocuteur. D'entrée de jeu la question d'Einstein va orienter le débat: Y a-t-il un moyen de libérer les hommes de la fatalité de la guerre ${ }^{11}$ ? Le problème de la guerre, va-t-il souligner, est devenu une question de vie ou de mort pour la civilisation, si l'on tient compte des progrès de la science moderne et de la facilité qu'auraient les hommes de s'exterminer mutuellement jusqu'au dernier. Tous les efforts déployés jusqu'à ce jour pour trouver une solution ont lamentablement échoué.

Pourquoi s'adresse-t-il à Freud ? Parce qu'il constate l'échec et l'impuissance de ceux qui ont à charge de s'occuper pratiquement et professionnellement de ce problème, c'est-à-dire les politiciens et les diplomates. En désespoir de cause, ceux-ci se tournent vers les hommes de science avec le souhait d'avoir leur avis mais aussi, probablement, avec l'espoir que la distance que garantit le travail scientifique puisse offrir une vision plus dégagée des problèmes de la vie.

Einstein commence par avouer son incompétence. Il ne peut que poser le problème, écarter les solutions les plus évidentes et laisser à Freud l'occasion d'éclairer la question sous l'angle de [sa] connaissance approfondie de la vie pulsionnelle humaine ${ }^{12}$. Voilà Freud convoqué comme expert de la vie pulsionnelle. Mais comme le souligne Pontalis dans une note de son article Actualité du malaise, cette convocation va plus loin :

Voici donc Freud, si longtemps méconnu ou rejeté comme simple chercheur, appelé à tenir la place du grand penseur, c'est-à-dire sommé de répondre non plus aux questions que son travail lui pose et dans les termes qui sont les siens mais à celles que le «temps présent» est censé poser et pour lesquelles il exige une réponse immédiate ${ }^{13}$.

16 Il semble évident, affirme Einstein, que des obstacles psychologiques rendent difficile ce combat contre la guerre; il demande à Freud de suggérer des méthodes éducatives plus ou moins étrangères à la sphère de la politique qui permettraient d'éliminer ces obstacles. Sa demande est pédagogique. De son côté, il avance une hypothèse sur les conditions d'une sécurité internationale. Il envisage de traiter l'aspect qu'il appelle extérieur, c'est-à-dire administratif ou organisationnel du problème. Il propose la création par les États - donc à partir d'un consensus international - d'une institution supranationale, une sorte de corps législatif et judiciaire dont la fonction serait d'arbitrer tout conflit qui surgirait entre les 
nations. Chaque nation s'engagerait alors à exécuter toute mesure que le tribunal considérerait nécessaire pour l'application de ses décrets.

Il fait une première constatation: la sécurité internationale implique le renoncement inconditionnel par les États à une partie de leur souveraineté au profit de l'institution supranationale.

Cependant on se heurte à un obstacle. Un tribunal est une institution humaine qui n'a pas à sa disposition la puissance pour exécuter ses verdicts; il s'expose ainsi à leur détournement sous l'effet de pressions extra-juridiques. Einstein part d'un fait avec lequel on doit nécessairement compter: droit et puissance sont indissociablement liés ${ }^{14}$. Les décisions juridiques sont d'autant plus proches de l'idéal de justice exigée par la communauté - au nom et dans l'intérêt de laquelle les verdicts sont prononcés - que cette dernière a la force de coercition pour faire respecter son idéal juridique. Nous sommes, donc, loin d'une telle institution supranationale, conclut-il. Cela reste aussi vrai encore aujourd'hui en 2007. Mais quelles forces s'opposent à la réalisation d'un tel programme ? Se transformant en psychologue, Einstein cherchera à cerner les puissants facteurs psychologiques qui paralysent les efforts qui tendent à assurer cette sécurité internationale. Il cernera d'abord le besoin de puissance, caractéristique de la classe dominante de tous les États qui manifeste son hostilité à toute limitation de la souveraineté nationale. Ce besoin politique de puissance va s'alimenter dans les activités d'un autre groupe dont les aspirations [sont] économiques et mercenaires, affirme-t-il. Ce groupe voit dans la guerre, dans la fabrication et le trafic des armes une occasion de favoriser ses intérêts particuliers et d'étendre son autorité personnelle. Comment est-il possible, demande-t-il, que la susdite minorité puisse mettre au service de ses désirs la masse du peuple qui, dans une guerre, ne peut que souffrir et perdre ${ }^{15}$ ? La minorité au pouvoir, la classe dominante, a la mainmise sur l'école, la presse et toutes les organisations religieuses. Par ces moyens, elle domine et dirige les sentiments de la grande masse et en fait le docile instrument ${ }^{16}$. Cette réponse, bien que juste, ne le satisfait pas pleinement. Elle engendre une nouvelle question : comment est-il possible que la masse se laisse enflammer par lesdits moyens jusqu'à la frénésie et au sacrifice de soi ${ }^{17}$ ? Einstein avance une nouvelle hypothèse qui va dans le même sens de la réponse que Freud apportera dans sa missive : en l'homme vit un besoin de hair et d'anéantir. En temps normal, il existe à l'état latent; cependant, dans des circonstances inhabituelles, il peut être réveillé avec une relative facilité et s'intensifier en psychose de masse. C'est sur cette question qu'il sollicite l'intervention de Freud, le grand connaisseur des pulsions humaines ${ }^{18}$. On ne peut pas ne pas évoquer ici le texte de 1915, Actuelles sur la guerre et la mort, qui répond déjà, à sa façon, à la question d'Einstein. Analysant l'idée de meurtre, Freud insiste sur le fait que la guerre nous dépouille des acquisitions de la civilisation, mettant à nu l'homme originaire qui est en nous. C'est précisément, écrit-il, commentant la sixième parole du Décalogue, l'accent mis sur le commandement: Tu ne tueras point, qui nous donne la certitude que nous descendons d'une lignée infiniment longue de meurtriers qui avaient dans le sang le plaisir au meurtre, comme peut-être nous-mêmes encore ${ }^{19}$. Les Lagers et les Goulags qui ont proliféré au siècle qui pourrait paraître le plus civilisé, le XXè siècle, permettent d'illustrer à souhait ces affirmations. Il est important de souligner que, pour Einstein, cette «psychose» n'est pas le fait de

ceux qu'on appelle incultes mais c'est bien plutôt précisément ce qu'on appelle «intelligence» qui succombe le plus facilement aux fatales suggestions de masse, parce qu'elle n'a pas coutume de puiser directement dans l'expérience de la vie, mais que la façon la plus commode et la plus achevée de la capter passe par la voie du papier impriméz ${ }^{20}$. 
Cette dernière hypothèse d'Einstein - le besoin de haine et de destruction - suscite dans son esprit une nouvelle question à l'adresse de Freud; c'est la question thérapeutique :

Y a-t-il une possibilité de diriger le développement psychique des hommes de manière à ce qu'ils deviennent davantage capables de résistance face aux psychoses de haine et d'anéantissement ${ }^{21}$ ?

Si Einstein n'a envisagé jusqu'à présent que les seuls conflits internationaux, c'est que le choix du conflit entre communautés humaines est, selon lui, délibéré; il obéit à des fins de démonstration; c'est en partant de cette forme-là, la plus typique mais aussi la plus cruelle, que l'on peut le mieux déceler la façon et les moyens d'éviter les conflits armés. Cependant il insiste, dans sa conclusion, sur le fait que cette pulsion agressive, ce besoin de haine et de destruction, s'exprime également dans les guerres civiles et dans la persécution des minorités. Il est présent dans toute relation humaine. Il attend de Freud qu'il développe, spécialement à la lumière de [ses] nouvelles connaissances, le problème de la pacification du monde $e^{22}$.

\section{Freud et la guerre}

\section{a/ Le meneur}

Il est cependant curieux de constater que les deux premières questions d'Einstein n'aient pas suscité chez Freud une association avec une œuvre écrite quelques dix années auparavant, 1921, Psychologie des foules et analyse du moi, essentielle à la réflexion sur la question de la guerre. Il y affirme que le social ne se dissocie pas de l'individuel pour constituer une strate à part du psychisme. Il est constitutif du psychisme. L'opposition entre la psychologie individuelle et la psychologie sociale est mise en question:

dans la vie psychique de l'individu pris isolément, écrit Freud, l'Autre intervient très régulièrement en tant que modèle, objet, soutien et adversaire, et de ce fait la psychologie individuelle est aussi d'emblée et simultanément une psychologie sociale, en ce sens élargi, mais parfaitement justifié23.

La psychologie sociale part de l'individu et s'interroge sur ce qui se passe dans sa psyché lorsqu'il est plongé dans une masse, quels types de forces cette foule va faire peser sur son fonctionnement psychique, et quelles modifications celui-ci sera contraint d'opérer devant la pression de ces forces ${ }^{24}$. Freud y développe à la fois une réflexion sur la nature de la masse, la formation des groupes et sur le chef, le Führer. Au cours des années où cette correspondance s'écrit, la question du Führer est capitale pour la compréhension de la catastrophe à venir.

Dans son analyse de l'hypnose, Freud fournit quelques éléments essentiels de réflexion. La relation hypnotique est une relation de masse à deux. La structure de la masse est complexe; l'hypnose en retient un seul élément qu'elle isole : le comportement de l'individu en foule envers le meneur ${ }^{25}$. Comparant l'hypnose à l'état amoureux, Freud écrit: même soumission humble, même docilité, même absence de critique envers l'hypnotiseur comme envers l'objet aimé. Même résorption de l'initiative personnelle; aucun doute, l'hypnotiseur a pris la place de l'idéal du moi [...] Cette dernière assertion reprend la formule qui résume l'état amoureux : l'objet est mis à la place de l'idéal du moi. L'hypnotiseur est l'objet unique, à côté de lui nul autre ne compte, ajoute-t-il. La relation hypnotique est un abandon amoureux illimité, la satisfaction sexuelle étant exclue ${ }^{26}$. Ce que l'hypnotiseur affirme et demande est vécu oniriquement par l'hypnotisé. Il est important de souligner d'abord que les situations de 
«masse» ne se définissent pas tant par le nombre de personnes qui y figurent que par le fait d'être régies par la fonction de l'idéal ${ }^{27}$; ensuite que les tendances sexuelles inhibées parviennent à créer des liens très durables entre les êtres humains dans la mesure où elles ne sont pas susceptibles de produire une pleine satisfaction, contrairement aux tendances sexuelles non inhibées qui, à travers la décharge, s'éteignent après la satisfaction. Pour durer, ces dernières doivent être intriquées à des composantes inhibées, c'est-à-dire purement tendres. Cette analyse permet à Freud d'expliquer à la fois le lien qui unit les individus dans la masse et celui qui les unit au chef; une masse ou une foule primaire, conclut-il, est une somme d'individus, qui ont mis un seul et même objet à la place de leur idéal du moi et se sont en conséquence, dans leur moi, identifiées les uns aux autres ${ }^{28}$.

Ce dont Freud nous parle, c'est de la dissolution de l'individu dans la masse qui se produira dans les années qui suivront, une véritable massification de l'individu qui disparaît en tant que tel à travers cette identification au meneur, posé comme idéal. Il s'agira d'une massification totalitaire. Cela est d'autant plus vrai que, contrairement aux «masses artificielles» que Freud étudie - l'Armée et l'Église -, des meneurs comme Hitler, Mussolini ou Staline ne se réclament d'aucun idéal transcendant, ils sont eux-mêmes l'incarnation de l'idéal. Comme l'écrit Hannah Arendt: la qualification majeure d'un leader de masses est désormais une infaillibilité éternelle; il ne peut jamais admettre d'erreur ${ }^{29}$. Elle ajoute plus loin que, dans le cas d'Hitler, le premier commandement. était: Le Führer a toujours raison. Nous sommes au cœur de la relation hypnotique que Freud décrit mais aussi au cœur de cette violence dont il sera question dans sa réponse à Einstein. En effet, la crédulité des masses est alors à son comble et le meneur peut leur faire croire les déclarations les plus fantastiques un jour, allant même, comme le fait encore remarquer Hannah Arendt, jusqu'à leur donner la preuve irréfutable du contraire, le lendemain. Loin d'abandonner leurs chefs, elles protesteront qu' [elles] avaient toujours su que la déclaration était mensongère, et [admireront] les chefs pour leur intelligence tactique supérieure ${ }^{30}$.

\section{b/ La communauté et l'individu}

Dans sa réponse à Einstein, Freud propose de substituer au couple droit et puissance, le couple droit et violence dont les termes, aujourd'hui antinomiques, se confondaient à l'origine. La question des rapports du droit et de la violence concerne principalement la réglementation des relations des hommes entre eux ${ }^{31}$. Il s'agit d'une des deux fins qu'il assigne à la Kultur, l'autre étant la protection de l'homme contre la nature, qui présuppose la domination des forces de la nature. Analysant les traits caractéristiques d'une culture, il écrit dans Malaise dans la culture:

peut-être commence-t-on à déclarer que l'élément culturel est donné avec la première

tentative pour régler ces relations sociales [...] qui concernent l'homme comme voisin, comme aide, comme objet sexuel d'un autre, comme membre d'une famille, d'un État ${ }^{32}$.

L'avenir d'une illusion soulignait déjà que de ces deux fins, c'est cette dernière qui provoque la plus profonde et la plus amère des insatisfactions face à la lenteur de ses «progrès» alors que la domination de la nature connaît des progrès constants.

Mais pourquoi cette substitution du terme de puissance ou de force par celui de violence ? Je penserais que Freud a en tête le pulsionnel. Cette violence est autant celle du sexuel que celle de la pulsion d'agression ou de destruction qui ne cessera, après la guerre de 1914 et le tournant de 1920, de le préoccuper. Déjà dans le mythe de la horde primitive dominée par le père tout-puissant, dans Totem et tabou, l'inceste, le meurtre et le 
cannibalisme apparaissent comme les désirs fondamentaux qui se dégagent de la pulsion. Cette violence ne s'exerce pas uniquement contre autrui, mais contre soi, si l'on se souvient que toute culture doive s'édifier sur la contrainte et le renoncement pulsionnel ${ }^{33}$. Il est vrai qu'en contrepartie la culture offre des dédommagements, tels les gains acquis dans la domination de la nature par les communautés humaines. Mais ces dédommagements sont-ils à la hauteur des renoncements ou ne sont-ils que des leurres ? Le jeu en vaut-il la chandelle ? Toujours est-il qu'ils ne font qu'intensifier la revendication pulsionnelle qui débouche sur une augmentation de l'hostilité de l'homme contre la culture qui, sans cesse, relance le processus meurtrier ${ }^{34}$.

De Totem et tabou à Pourquoi la guerre? en passant par Psychologie des foules et analyse du moi, Le malaise dans la civilisation nous retrouvons une même idée : le lien indissociable entre l'individu et la communauté. Cette idée constitue le fil rouge qui permet de lire la lettre de Freud et d'en articuler les différents thèmes. Qu'est-ce que Freud entend par communauté ? La réunion d'une majorité d'êtres faibles qui se constitue contre l'individu le plus fort, faisant prévaloir sa violence contre lui. La vie en commun des hommes n'est rendue possible, écrit Freud, que si se trouve réunie une majorité qui est plus forte que chaque individu et qui garde sa cohésion face à chaque individu ${ }^{35}$. Cette violence collective, dont la communauté revendique le monopole contre l'individu qui voudrait ou pourrait l'attaquer, est ce que nous appelons droit. La puissance de cette communauté, continue Freud, s'oppose maintenant en tant que «droit» à la puissance de l'individu qui est condamnée en tant que violence brute. Il ajoute : ce remplacement de la puissance de l'individu par celle de la communauté est le pas culturel décisif ${ }^{36}$. L'essence de ce remplacement consiste dans la limitation des possibilités de satisfaction des membres de la communauté, alors que la puissance de l'individu isolé, à l'état de nature, était illimitée.

Dans sa lettre, Freud affirme, de façon un peu lapidaire à mon sens, que la cohésion de la communauté dépend de deux facteurs: la contrainte de la violence et les liens de sentiments - les identifications - entre les membres du corps communautaire. Il ajoute que si l'un des facteurs manque, l'autre peut éventuellement maintenir la communauté. Psychologie des foules et Malaise dans la culture jettent un éclairage sur les mécanismes en jeu en analysant la constitution d'une communauté.

Cette dernière a comme conditions nécessaires, comme nous l'avons vu, d'une part la présence d'un chef et d'autre part les relations amoureuses(en termes neutres: liens sentimentaux $)^{37}$ qui vont unir les membres du groupe au chef. C'est Éros qui crée ces liens et les consolide :

(...) la foule doit manifestement sa cohésion à un pouvoir quelconque. Mais à quel pouvoir pourrait-on attribuer cet exploit si ce n'est à l'Éros à qui le monde entier doit sa cohésion ${ }^{38}$ ?

31 Éros, cependant, ne saurait régner seul; la haine est aussi nécessaire, elle fait partie de la pulsion de mort qui s'exprime sous forme de pulsion de destruction tournée vers l'extérieur et amplifiée du fait de son inscription dans la communauté. S'il n'est pas facile aux hommes de renoncer à satisfaire ce penchant à l'agression qui est le leur ${ }^{39}$, comment cette haine, va-t-elle trouver à s'exprimer ? La communauté, pour subsister, va se créer des ennemis extérieurs auxquels elle fera la guerre mais aussi des ennemis intérieurs qui prendront la forme du bouc émissaire ou celle de la guerre civile. Il est toujours possible de lier les uns aux autres dans l'amour une assez grande foule d'hommes, si seulement il en reste d'autres à qui manifester de l'agression ${ }^{40}$.

L'histoire de Juifs et celle de la Russie communiste servent à illustrer autant le mécanisme de formation et de permanence des communautés que l'expression de la haine. Freud 
rappelle comment l'instauration de l'amour universel comme fondement de la communauté chrétienne par l'apôtre Paul a eu pour conséquence inévitable l'extrême intolérance du christianisme à l'égard de ceux qui n'en faisaient pas partie, particulièrement des Juifs.

Quant à la Russie, la tentative d'édifier [...] une nouvelle culture communiste trouve son support psychologique dans la persécution des bourgeois. Il conclut par cette question fort pertinente, surtout lorsqu'on la considère après-coup : on se demande seulement avec inquiétude ce que les Soviets entreprendront une fois qu'ils auront exterminé leurs bourgeois ${ }^{41}$. Poursuivant sa réflexion, dans sa réponse à Einstein, il dénonce l'illusion des bolchevicks qui espèrent pouvoir faire disparaître l'agression humaine en garantissant la satisfaction des besoins matériels et en instaurant par ailleurs l'égalité entre ceux qui font partie de la communautét ${ }^{2}$. Cette dénonciation se situe dans le contexte où l'idéal rousseauiste d'une nature riche et abondante où la vie s'écoule dans la douceur et l'harmonie est critiqué, mais aussi cette illusion est confrontée à l'usage de la haine que font les communistes pour maintenir la cohésion de leur communauté. Je ne puis qu'évoquer rapidement un texte contemporain de cette lettre et qui doit être lu en même temps qu'elle, la trente-cinquième conférence, Sur une Weltanschauung, des Nouvelles conférences d'introduction à la psychanalyse ${ }^{43}$ où Freud analyse conjointement les illusions religieuse, philosophique et marxiste. Au-delà d'illusions spécifiques, ce que Freud démonte ici ce sont les mécanismes de l'illusion politique comme il l'avait fait en 1927 avec l'illusion religieuse. Si la psychanalyse n'a pas de Weltanschauung spécifique, comme il le montre dans ce texte, c'est bien parce son travail en est un de démystification. Elle travaille dans le désillusionnement.

Comme nous pouvons le constater par ces exemples ainsi que par les mécanismes de sa constitution, la communauté ne fait disparaître ni la violence ni la guerre. Pourquoi ? Parce qu'une communauté contient dans son sein des éléments de puissance inégale (hommes, femmes, parents, enfants, etc.) qui font que le droit de la communauté devient alors l'expression des rapports de puissance inégau ${ }^{44}$. Autrement dit, la violence est légalisée. Il en résulte que les lois sont faites par et pour les dominants. Une lutte va s'instaurer alors au sein de la communauté qui sera source à la fois de désordre et de progression en matière de droit. Les dominants voudront faire des lois qui les favorisent ou se situer en dehors d'elles et ainsi revenir du règne du droit au règne de la violence. Les opprimés, de leur côté, vont lutter pour se procurer plus de puissance et pour voir ces modifications reconnues dans la $l o i^{45}$. Leur mouvement va dans le sens contraire : réclamer le passage d'un droit inégal vers un droit égal pour tous. Cependant la classe dominante n'est pas prête à accepter ces modifications. Il s'ensuivra l'insurrection, la guerre civile, la suppression temporaire du droit. On en vient [...] à de nouvelles et violentes épreuves de force, à l'issue desquelles un nouvel ordre juridique est instituét ${ }^{46}$. La guerre semble donc inévitable. Quels moyens Freud propose-t-il pour la combattre ? Il suggère particulièrement deux voies indirectes que je voudrais rapidement analyser. La première consiste à contrer le pouvoir de la pulsion de destruction par son antagoniste, l'Éros. Tout ce qui instaure des liaisons de sentiment parmi les hommes, écrit-il, ne peut qu'agir contre la guerre ${ }^{47}$. Ces liaisons sont de deux sortes : d'une part, des relations comme celles qui s'établissent avec un objet d'amour, même sans intentions sexuelles; d'autre part, le lien sentimental qui procède de l'identification. Autrement dit, la constitution d'une communauté de plus en plus large. Or nous savons que la communauté ne maintient sa cohésion qu'à condition de construire des ennemis extérieurs ou intérieurs contre lesquels elle détourne sa haine. Freud peut-il croire en sa solution? 

aveu d'idéal qu'il avait déjà énoncé dans la $35^{\mathrm{e}}$ conférence des Nouvelles conférences d'introduction à la psychanalyse, la dictature de la raison sur la vie pulsionnelle; elle reposerait sur la formation d'une élite d'êtres humains à la pensée autonome, qui ne puissent pas être intimidés et qui luttent pour la vérité. Mais l'empire de l'État et de ses pouvoirs et l'interdit de pensée édicté par l'Église rendent cette voie impraticable et utopique. Comment Freud peut-il croire d'une part à l'éducation de ce genre de meneurs alors qu'il a si bien cerné le profil du meneur dans Psychologie des foules comme je l'ai montré plus haut ? D'autre part, une dictature de la raison sur la vie pulsionnelle est-elle possible? La raison peut-elle atteindre un tel degré d'autonomie que la vie pulsionnelle ne l'infiltre plus et ne la dérègle plus ? Une telle autonomie, si elle était possible, serait-elle pour autant souhaitable? Que serait un être humain qui fonctionnerait selon cette dictature sans un grain de folie?

Chaque mot étonne, chacun éveille un paradoxe, voire une énigme, écrit Marie Moscovici. L'appel à la raison chez celui qui a exposé et fait vivre, dans la théorie et la pratique, le pouvoir de l'inconscient, le règne de l'étrange et de l'insensé. Le recours à une dictature, chez l'homme qui a récusé toutes les soumissions de la pensée $e^{48}$.

Si Freud n'a pas vécu la catastrophe, ses analyses laissent pressentir le pire et montrent que la barbarie est au cœur de la Kultur. C'est ce qu'Adorno soulignera fortement dans Éduquer après Auschwitz où il pose comme exigence première de l'éducation, qui n'a pas à se justifier, qu'Auschwitz ne se reproduise pas. Plus jamais d'Auschwitz, un nouvel impératif catégorique comme il le nomme dans la Dialectique négative. Dans leur état de nonliberté, Hitler a imposé aux hommes, écrit-il, un nouvel impératif catégorique: penser et agir en sorte que Auschwitz ne se répète pas, que rien de semblable n'arrive ${ }^{49}$. C'est une nécessité. Il constate cependant qu'on ne s'en est pas préoccupé davantage à ce jour et que l'on n'est guère conscient de cette nécessité. Cela montre bien que cette monstruosité n'a pas pénétré profondément dans le niveau de conscience et d'inconscience des hommes ${ }^{50}$. Pour lui, la barbarie persiste. La civilisation engendre l'anticivilisation et la renforce ${ }^{51}$, conclut-il de sa lecture de Malaise dans la culture et de Psychologie de foules et analyse du moi. Il ajoute : si la barbarie s'inscrit au principe même de la civilisation, il semble désespéré de vouloir s'y opposer ${ }^{52}$, rejoignant ainsi la question finale de Freud dans Malaise dans la culture: le destin de l'espèce humaine dépend de la capacité du développement culturel maitriser la perturbation que la pulsion d'agression apporte à la vie en commun.

\section{NOTES}

1. Freud S. (1956 [1950]), La naissance de la psychanalyse, Paris, PUF, Bibliothèque de psychanalyse, p.186.

2. Freud S. (1908 [1970]), La morale sexuelle civilisée et la maladie nerveuse des temps modernes in La vie sexuelle, Paris, PUF, p.45

3. Sous le titre, Freud et la question de la guerre, j'ai analysé cet écrit. Cette analyse devrait paraître à l'automne 2007 dans la revue Topique.

4. Zweig S. (1944 [1993]), Le monde d'hier, souvenirs d'un européen, Paris, Belfond. 
5. Ibid, p.20

6. Pour la distinction entre crise et malaise, je renvoie à l'article remarquable de J.B.Pontalis (1988) Actualité du malaise in Perdre de vue, Paris, Gallimard, folio essais, p.23-46.

7. Kahn L. (2005), Faire parler le destin, Paris, Klincksieck, p.191.

8. Freud, S. (1939 [1986]), L'Homme Moïse et la religion monothéiste, Trois essais, Paris, Gallimard, Connaissance de l'inconscient, p. 132.

9. The standard edition of the complete psychological works of Sigmund Freud, volume XXII, 1932-1936, London, The Hogart Press and the Institute of psycho-analysis, p.197-198.

10. Par exemple dans Résultats, idées, problèmes II, PUF, on est surpris de constater que seule figure la réponse de Freud.

11. Freud S [1933 (1995)], Pourquoi la guerre? in CEuvres complètes Psychanalyse, vol XIX, 1931-1936, PUF, p.65.

12. Ibid, p. 65.

13. Perdre de vue, p.27, note 2 .

14. Ibid, p.66.

15. Ibid, p.67.

16. Ibid, p.67.

17. Ibid, p.67.

18. Ibid, p.67.

19. Actuelles sur la guerre et la mort, p.151

20. Ibid, p.67-68. Cette affirmation d'Einstein est à rapprocher de cette autre affirmation d'Adorno et Horkheimer dans La dialectique de la raison, Fragments philosophiques, nrf, Gallimard, 1974, p.16: La mystérieuse disposition qu'ont les masses à se laisser fasciner par n'importe quel despotisme, leur affinité autodestructrice avec la paranoïa raciste, toute cette absurdité incompréhensible révèle la faiblesse de l'intelligence théorique actuelle

21. Ibid, p.67.

22. Ibid, p.68.

23. Freud, S (1921 [1987], Psychologie des foules et analyse du moi in Essais de psychanalyse, Petite Bibliothèque Payot, nouvelle traduction, p.123

24. p.24

25. Psychologie des foules, p. 180

26. Ibid, p.179.

27. Scarfone, Dominique (1999), Oublier Freud ?, Montréal, Les Éditions du Boréal, p.173.

28. Psychologie des foules, p.181

29. Arendt, Hannah (1948 [2002]), Le totalitarisme in Les origines du totalitarisme, Quarto, Gallimard, p.666.

30. Le totalitarisme, p.709

31. Le malaise dans la civilisation, p.32.

32. Ibid, p.38.

33. Freud S (1927 [1995]), L'avenir d'une illusion, Paris, Quadrige, PUF, p.7

34. Kahn, Laurence (2004), Fiction et vérité freudiennes, Entretiens avec Michel Enaudeau, Paris, Balland, p.239

35. Le malaise dans la civilisation, p.38

36. Ibid, p.38.

37. Psychologie des foules et analyse du moi, p.152.

38. Ibid, p.152.

39. Freud, S (1929 [1995]), Le malaise dans la culture, Quadrige, PUF, p.56

40. Ibid, p.56.

41. Ibid, p.57.

42. Ibid, p.78 
43. Freud S. (1933 [1984]), Nouvelles conférences d'introduction à la psychanalyse, Connaissance de l'inconscient, nrf, Gallimard, pp 211-243.

44. Ibid, p.72.

45. Ibid, p.72

46. Ibid, p.72

47. Ibid, p.78

48. Moscovici Marie (1991), Il est arrivé quelque chose, Paris, Payot, p.225.

49. Adorno W. Theodor (1966 [2001]), Dialectique négative, Paris, Petite Bibliothèque Payot, p.442.

50. Adorno W. Theodor (1963 [2003]), Modèles critiques, Paris, Payot, p.235.

51. Adorno W. Theodor, Modèles critiques, p.235.

52. Ibid, p.236.

\section{AUTEUR}

\section{DAVID BENHAIM}

Philosophe, Psychanalyste membre de la Société canadienne de psychanalyse et de la Société psychanalytique de Montréal 